\title{
XRD Evaluation of Relative Mechanical Strength and Irradiation Resistance of Synthetic Diamond
}

\author{
E. M. Shishonok, V. G. Luhin \\ Belarusian State University of Technology, Minsk, Republic of Belarus \\ Email: eshishonok@tut.by
}

Received 29 October 2015; accepted 10 December 2015; published 17 December 2015

\begin{abstract}
We continue here our previous work where SD powders were significantly strengthened by irradiation with electrons of lower energy under smaller dose. Previous results were obtained from the crushing strength analysis, no XRD was applied. In present work, powders of synthetic diamond with low strengthwere sorted on sets with different grain size. As established, the sets had various crushing strengths and morphology. They were irradiated with high energy electrons $(6.5 \mathrm{MeV}, \mathrm{D}$ $=2 \times 10^{19}$ and $\left.\mathrm{D}=6 \times 10^{19} \mathrm{~cm}^{-2}, \mathrm{~T}_{\text {irr }}=450 \mathrm{~K}\right)$ and analyzed using XRD $\left(\mathrm{CuK}_{\alpha}\right)$ before and after irradiation. Nonlinear dependences $a(\Theta)=f\{R(\Theta)$, where $a(\Theta)$ is lattice constant and $R(\Theta)$ is Raily function, and the discovered extra-splits (additional to $\alpha_{1}-\alpha_{2}$-doublets on $\mathrm{CuK}_{\alpha}$ ) of basic peaks in XRD patterns from the SD sets, testified that crystal lattice of diamond in sets was variously distorted, like of $\mathrm{cBN}$ doped with rare earth elements. As established, the first irradiation led to decreasing distortions, the more significantly the higher initial strength of the set. The second irradiation produced softening and increasing distortions of crystal lattice of diamond, the more effectively the less initial strength of diamond. XRD allows indirectly to presort synthetic diamond off the material with critically low relative mechanical strength as well as evaluate resistance of diamond crystal lattice against heavy irradiation and other external impacts.
\end{abstract}

\section{Keywords}

Synthetic Diamond, Crushing Strength, Electron Irradiation, XRD, Crytsalline Distortion

\section{Introduction}

Diamond as super hard material is widely used in industrial applications. As wide band gap semiconductor $\left(\mathrm{E}_{\mathrm{g}}=\right.$ $5.5 \mathrm{eV}$ ) diamond in natural (ND) and synthetic (SD) forms is used in electronic devices. Only cubic boron nitride $\left(\mathrm{E}_{\mathrm{g}}=6.4 \mathrm{eV}\right)$ as the closest analogue of diamond which exists only in synthetic (cBN) form, is superior to diamond in thermal, radiative and chemical stability. Both synthetic materials can be synthesized under high pressure-high temperature (HPHT) conditions.

A radiation resistance of electronic devices should be guaranteed by stability of their functional materials when the devices should be set into extremely harsh radiation surrounding. As to diamond, degradation of its 
mechanical strength is the direct and critical criterion for link establishment with radiation resistance of diamond. The present work is aimed on development of the indirect evaluation method of SD relative mechanical strength and the material's radiation resistance.

Due to defects and impurities, characteristics (e.g., thermal conductivity, mechanical strength, radiative resistance and other macro-properties) of synthetic diamond (SD) and cBN are lower than that expected. Defects in diamond have been widely investigated by use of different spectroscopic methods (PL, CL, EPR, Raman, IR). A lot of optically active and paramagnetic defects in natural and synthetic diamond were discovered and justified, and perfectly systemized (e.g. [1] [2]). SD has appeared as less expressive in number of the defects as compared to the natural one. To the present time, it is precisely established that nitrogen as the main impurity in diamond, variously influences the material's basic functional properties in dependence on form of nitrogen inclusions and its concentration in diamond. Additional imperfections in SD are expected due to catalysts atoms and doping impurities of large sizes, which are incorporated into SD crystal lattice during HPHT synthesis. As shown in [3], ferromagnetic impurities can be introduced into SD powders of low strength in larger concentration than nitrogen, and the non-uniform distribution of the impurities in crystal lattice of SD results in variation of mechanical strength of the material.

Since 1985, cBN has been investigated intensively using luminescence methods [4]. It has been established that like SD, cBN doesn't have many optically active defects even though the material includes noticeable concentrations of doping impurities.

The question arises about pretesting SD of average quality on stability of its crystal lattice, if the material contains impurities or is subjected to various external impacts. Should we apply direct measurements of mechanical strength to a functional SD, which can crush the latter in some cases?

XRD analysis allows to define a state of crystal lattice of SD and cBN. As recently discovered by XRD, cBN crystal lattice can be variously distorted by introducing rare earth ions $\left(\mathrm{RE}^{3+}\right)$ [5] [6]. Even intrinsic boron of large concentration in cBN leads the latter to crystalline distortion [7].

The present work followed our investigations on $\mathrm{cBN}: \mathrm{RE}^{3+}$. We had kept in mind that SD crystal lattice of low strength would have to possess distortions like a single-phase crystal lattice of $\mathrm{cBN}: \mathrm{RE}^{3+}$.

We distinguish here effects of strains of diamond crystal lattice from its crystal distortions. It is well known, that the simplest type of strain is a uniform dilatation. If a sample is strained isotropicly, there is a small uniform change in lattice parameter. For an uniform dilatation, the sharp peaks of XRD pattern of the sample shift in position as strong as the Bragg angle increases. In the case of a non-uniform strains, the sharp peaks broaden. In both cases the peaks stay of symmetric shape.

A lot of publications are devoted to these phenomena in SD and ND due to nitrogen impurities in the material. We refer to [8], where authors by use of precise diffractometry, investigated (440) reflections in XRD pattern from different faces of pure single crystal of synthetic diamond. Different concentrations of dispersive nitrogen $\left\{\mathrm{N}=10^{6}-(88 \pm 7) \times 10^{6} \mathrm{~cm}^{3}\right\}$ were obtained on different faces, and symmetric shape of the reflections was mentioned. We say here about the non-uniform dilatation.

On the other hand, distortion of cubic crystal lattice leads to lowering its cubic symmetry. In XRD patterns from the distorted cubic crystal lattice appear extra-splits of basic peaks of cubic structure. The splits follow the appearance of new (hkl) planes $d$-spacing caused by breaking the rule for the multiplicity factor $m$. Peaks of XRD pattern of perfect cubic crystal lattice do not split by use of monochromatic roentgen irradiation [9] [10]. Again referring to [8], we cannot expect there any distortion of SD crystal lattice.

In our previous work [11], we irradiated SD industrial powders with $4 \mathrm{MeV}$ electrons within doses $\mathrm{D}=1 \times$ $10^{17}-2 \times 10^{18} \mathrm{~cm}^{-2}$ in vacuum and $\mathrm{CO}_{2}$ atmosphere. It was established, that the sufficient strengthening of SD grains as obtained starting from the dose of $\mathrm{D}=3 \times 10^{17} \mathrm{~cm}^{-2}$ and more effectively after irradiation in the atmosphere. In present work we increase energy and dose of irradiating electrons in order to establish the threshold for electrons dose which leads to breaking SD strength. We try to do it without direct strength's measurements. We try to develop representations about natural connection between relative strength of synthesized SD powders and peaks' shape in their XRD patterns. On the basis of the representation, we try to make judgment about relative strength of irradiated SD powders and even about strength of SD of any morphological form in coming work.

\section{Experimental Details}

Low strength SD industrial powders were synthesized under HPHT conditions using NiMn catalyst. Powders 
were sorted onto sets with grain sizes 63/50, 100/80, 160/100, 200/160 $\mu$ using standard sieves analysis. Concentrations of dispersive nitrogen in the SD sets varied within $\sim(1.8-2.4) \times 10^{18} \mathrm{~cm}^{3}$, that was estimated from ESR analysis. Electron irradiation of SD sets using $6.5 \mathrm{MeV}$ electrons $\left(\mathrm{D}=2 \times 10^{19}\right.$ and $\left.\mathrm{D}=6 \times 10^{19} \mathrm{~cm}^{-2}\right)$ in two rounds at $T=450 \mathrm{~K}$ was performed under vacuum condition. SEM analysis was applied to visualize morphological features of SD grains in the sets. An average strength of each SD set was measured using standard grains' crushing method. Two industrial parties of the same sets were investigated which showed very close results.

XRD patterns from the SD sets before and after irradiation were taken using D8 Advance Bruker AXS instrument on $\mathrm{CuK}_{\dot{\alpha}}$ radiation. For measurements, equal masses of powders were placed into standard cuvettes. The rotation option of samples was used to avoid random errors and to increase and consider contribution of all crystallographic planes of $\{h k l\}$ multiplicity into each $h k l$ reflex of XRD patterns during their recording with step of $0.01 \% \mathrm{~min}$. XRD patters records of the separate set were carried out repeatedly.

Computer program was used to decompose asymmetric peaks of XRD patterns on minimal number of Gaussian components, package of which could give calculated peaks with minimal deviation from the experimental peaks. It was taken into account that intensities ratio of $\dot{\alpha}_{1}$ - и $\dot{\alpha}_{2}$-components in $\mathrm{CuK}_{\alpha}$-doublet is equal two and the components have identical half-widths.

\section{Results and Discussion}

As seen from Figure 1(a) and Figure 1(b) the morphology of SD grains is the worse the lower strength of grains, and the 100/80 set possesses the maximal crushing strength. The most perfect morphology and strength are characteristic for crystallization centers of SD which appear in the most equilibrium thermodynamic conditions and when catalyst atoms (Ni, Mn) are near these centers. XRD patterns from all SD sets were represented by (111), (220), (311), (331) basic peaks related to diamond. The peaks were of various intensities for different SD sets as compared to natural diamond standard (Figure 1(c), e.g.). It was assumed, that SD could be textured or its crystal lattice was distorted [9] [10].
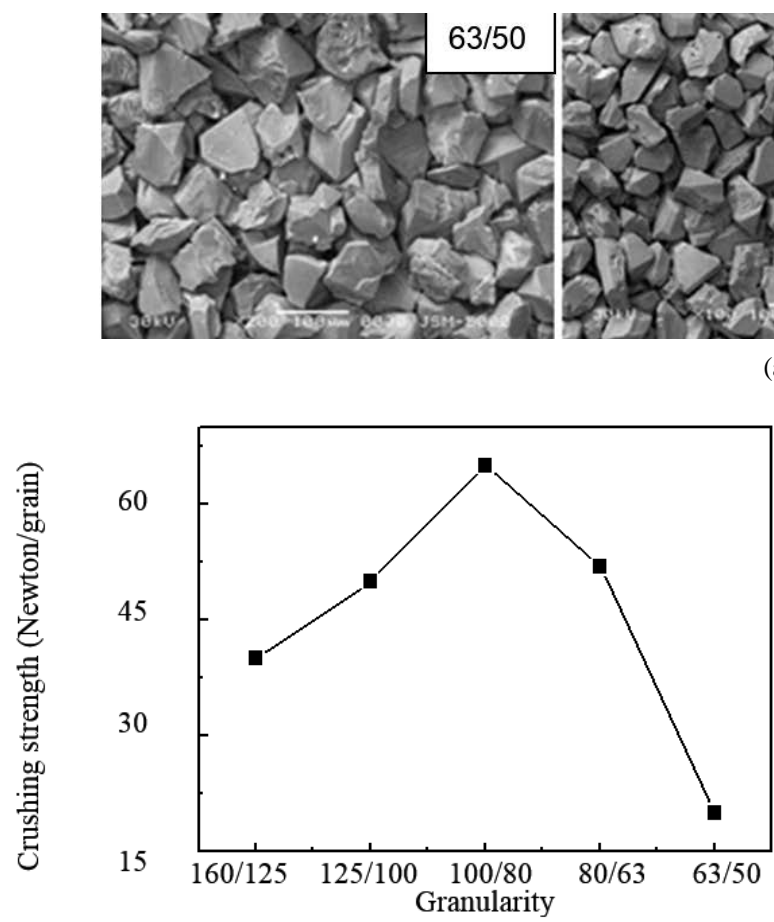

(b)

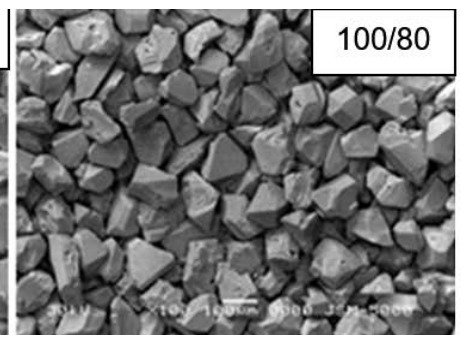

(a)
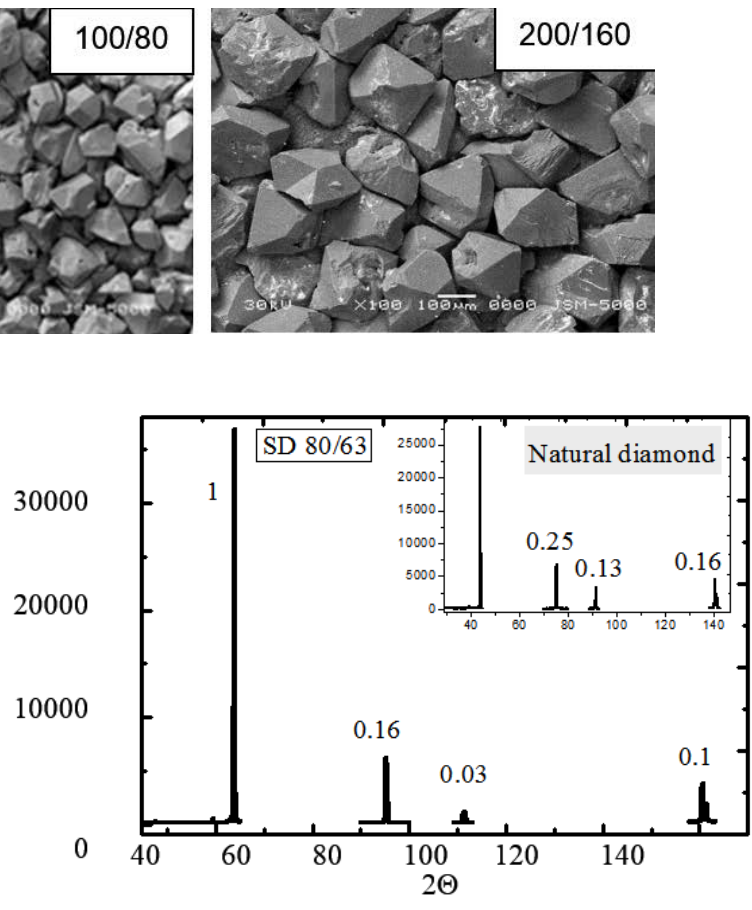

(c)

Figure 1. SEM images of SD sets, length of white line is equal $100 \mu \mathrm{m}$ (a); mechanical (crushing) strength of SD sets (Newton/grain) in dependence on granularity of the SD sets (b); XRD pattern from the 80/63 SD set, in the inset-of natural diamond standard, values of intensities are labeled (c). 
It is known that for a perfect crystal lattice, dependence of $a(\Theta)=f\{R(\Theta)\}$ is linear, where $a(\Theta)$ is a lattice constant and $R(\Theta)$ is Raily function, both calculated for each peak of XRD pattern from a perfect crystal lattice. If not, the crystal lattice is distorted [5] [7]. As established in present work, the dependences $a(\Theta)=f\{R(\Theta)\}$ are variously nonlinear for all SD sets. It is clear, that lattice constant of SD crystal lattice before irradiation, which had been calculated from each peak of XRD patters of the SD sets, changes non-regularly with increasing the Bragg angle. This distinguishes our case from non-uniform strains of SD crystal lattice in [8]. Irradiation of SD sets with $6.5 \mathrm{MeV}$ electrons $\left(\mathrm{D} \sim 2 \times 10^{19} \mathrm{~cm}^{-2}\right)$ led to changes of the nonlinear character of dependences $a(\Theta)=f\{R(\Theta)\}$ for investigated SD in the sets. They became almost linear, the more linear the higher initial strength of a set. Mostly due to increase of interplanar distance $d_{111}$. Figure 2(a) shows the dependences for the 100/80 set before and after first irradiation. The crystal lattice of SD after the first irradiation has became more perfect because its distortions decreased, possibly due to impurities redistribution into thermodynamically more favorable positions. As follows from the present work, the 100/80 set more evidently has enhanced the perfection of SD crystal lattice.

The dependences $a(\Theta)=f\{R(\Theta)\}$ for irradiated SD (in the 100/80 and 200/160 sets) under the summarized dose of $\mathrm{D} \sim 8 \times 10^{19} \mathrm{~cm}^{-2}$ and for non-irradiated SD (in the 100/80 set), are represented in Figure 2(b). As seen from the figure, lattice constant of SD in the sets after the second irradiation decreases non-regularly in dedependence on Raily function (or with increase of the Bragg angle). This result may indirectly indicate decreasing mechanical strength of SD set, the stronger the less its initial mechanical strength. The most suitable explanation for that is creating great number of vacancies in SD crystal lattice under the heaviest irradiation which led to significant softening crystal lattice of SD in the sets, and even as compared to the initial ones. It is clear that strengthening crystal lattice of investigated SD cannot be endless with increase of irradiation dose.

The obtained results were confirmed by discovering extra-splits of basic peaks of XRD patterns from the SD sets before and after irradiations, which are complementary to $\alpha_{1}-\alpha_{2}$-doublets [6] [12] [13]. First, some extrasplits could be visibly discovered (Figures 3(a) and Figures 3(b) in the left insets). Secondly, after recording XRD patterns using the rotation option the results were refined by decompositions of asymmetric basic lines onto Gaussians using computer program if it was possible (Figure 3).

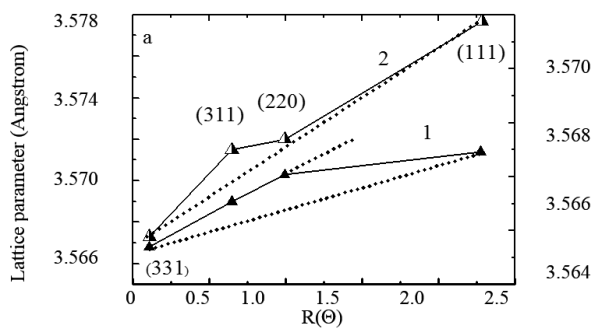

(a)

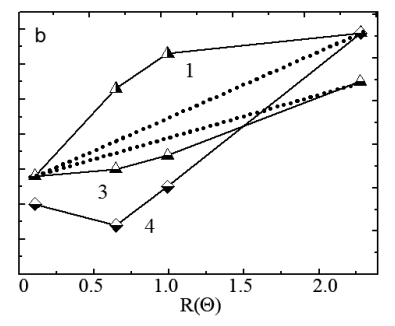

(b)

Figure 2. Dependences of SD lattice parameter (in Angstroms) on Railyfunction for the 100/80 SD set before (1) and after irradiation with dose D $\sim 2 \times 10^{19} \mathrm{~cm}^{-2}$ (2) (a); for the 100/80 SD set before and after irradiation $(1,3)$ and for the 200/160 SD set (4) after irradiation with summarized dose of $\mathrm{D} \sim 8 \times 10^{19} \mathrm{~cm}^{-2}$ (b). Rotation option is applied. Dot lines represent a possible variant for a perfect crystal lattice of SD.

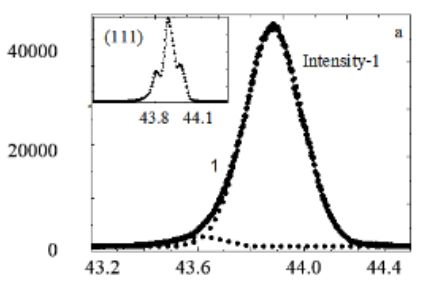

(a)

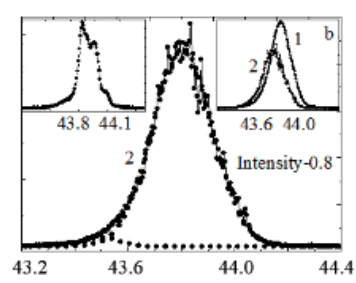

(b)

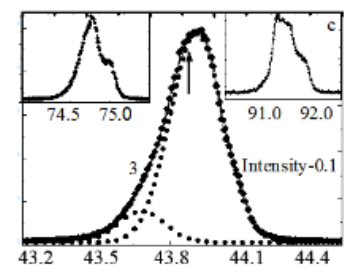

(c)

Figure 3. Peaks (111) in XRD patterns of the 100/80 SD set before irradiation, in the inset-without the rotation option (a); after the first irradiation, in the left inset-without the rotation option, in the right inset-as compared to the non-irradiated set (b); after the second irradiation, in the insets-shapes of (220) and (311) peaks (c). Decompositions into Gaussians are shown in dot lines. The arrow (c) indicates the maxima position for the non-irradiated SD set. 
As mentioned above, the extra-splits in XRD patters of cubic crystal structure are the proof that this cubic structure is distorted. The less number of additional peaks, the higher symmetry of distortions (tetragonal, trigonal and so on). Relative intensities and angular positions of additional peaks in XRD pattern as compared to basic peaks, are correlated with quantitative features of distortions.

In this paper, we would not intend to do evaluation about types of distortion of SD crystal lattice, like we did for cBN. We try to make some general conclusions which are in correspondence with basic purpose of our work. Therefore, all peaks in XRD patterns of investigated SD sets are not represented. It is also clear, that we should use the rotation option to investigate SD powders with large grain sizes.

Figure 3(a) shows that asymmetrical peak (111) in XRD pattern from the 100/80 SD set, is initially split into basic and additional peaks (non-resolved $\alpha_{1}-\alpha_{2}$-doublets). After the first irradiation, the additional peak of smaller intensity and width and peak's angle position, testify that number of equal interplanard ${ }_{111}$-spacings increased and the factual random distortion decreased, but the actual mathematical value of distortion is increased (e.g. $\Delta \gamma$-for trigonal distortion, $\gamma=90^{\circ}$ for cubic crystal lattice) (Figure 3(b)). After the second irradiation, the broad additional peak of high intensity and peak's angle position, testify that number of equal interplanar $d_{111}$-spacings decreased and the factual random distortion sufficiently increased, but the actual mathematical value of distortion is decreased (Figure 3(c)). All peaks in XDR pattern of the 100/80 set behaved themselves with tendencies which are similar to the behavior of peak (111) in compliance with Figure 2. Angular positions of all peaks are being changed. They acquire more symmetrical shape after the first irradiation and became more asymmetrical due to enhancing intensities of additional peaks after the second irradiation. We have supposed here that type of distortion in not changed.

Figure 4 represents peaks (111), (220) and (311) of XRD pattern from the 200/160 SD set after the first and the second irradiation. The set contained large grains which grow very quickly and initially had the lowest crushing strength. Intensities of peaks are much lower than that of the 100/80 SD set. It means that planes (hkl) are far from structural perfection. We can compare the intensities, because in measurements equal masses of powders were used. After the first irradiation, the remarkable additional peak within peak (111) testifies that are there remarkable distortions of crystal lattice of SD represented by the 200/160 set as compared to the 100/80 set. Peak (220) cannot be resolved only into perfect $\alpha_{1}-\alpha_{2}$-doublet. Peak (311) has complex structure and visually consists of three $\alpha_{1}-\alpha_{2}$-doublets (two of them represent two additional lines). The second irradiation leads to softening crystal lattice of SD within the 200/160 set, because all peaks in its XRD pattern shift to the range of large $2 \Theta$-angles, like it happened with all sets more or less. Besides that, peaks became more structured fvery well illustrated by peak (220)\}, as compared to the $100 / 80$ set. In general case, this would mean that symmetry of distortions of cubic crystal lattice is lowered. This is the proof that diamond crystal lattice within the 200/160 set is closer to degradation, than that of the 100/80 set.

\section{Conclusions}

Industrial powders of synthetic diamond of low strength were sorted into sets of different grain size with various crushing strength and morphology. The SD sets were investigated by XRD using the rotation option of samples. Nonlinear dependences $a(\Theta)=f\{R(\Theta)$, where $a(\Theta)$ is a lattice constant and $R(\Theta)$ is Raily function, and the discovered extra-splits (additional to $\alpha_{1}-\alpha_{2}$-doublets on $\mathrm{CuK} \alpha$ ) in XRD patterns from SD sets, testified that crystal lattice of diamond in sets was variously distorted. It looks like the materials represent variously disordered solid
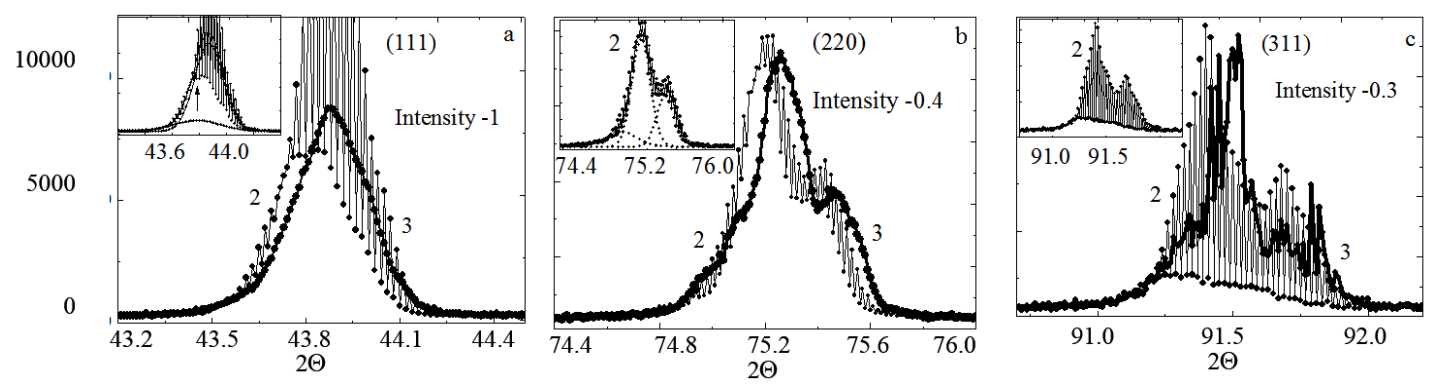

Figure 4. Peaks (111), (220) and (311) in XRD patterns from the 200/160 set after the first (2) and the second irradiation (3). In the insets peaks after the first irradiation and decomposed into Gaussian 
solutions of impurities in diamond. The higher degree of disordering of SD crystal lattice, the lower strength of the corresponding SD set. As established from transformation of the dependences and of the extra-splits, irradiation of the sets with electrons of higher energy and in larger dose $\left(6.5 \mathrm{MeV}, \mathrm{D}=2 \times 1019 \mathrm{~cm}^{-2}\right)$ as compared to our previous experiments, still lead to SD strengthening, the more effectively the higher initial strength of the SD set. Applying the total dose $\mathrm{D}=8 \times 10^{19} \mathrm{~cm}^{-2}$ leads to softening and increasing distortions of SD crystal lattice, the stronger the less initial strength of the SD set.

Thus, XRD analysis allows to presort synthetic diamond off the material with critically low relative mechanical strength as well as evaluate resistance of diamond against irradiation and other external impacts.

\section{References}

[1] Zaitsev, A.M. (2010) Optical Properties of Diamond: A Data Handbook. Springer Berlin Heidelberg.

[2] Davies, G. (1994) Properties and Growth of Diamond. Run Press Ltd., Exeter.

[3] Bogatirova, G.P., Maevski, V.B., Ilnitskaja, G.D., et al. (2006) Formation of the Contact Interaction Zone of Nickel Alloy with Carbon Surface. Superhard Materials (Ukraine), 4, 36-39.

[4] Shishonok, E.M. (2009) Cubic Boron Nitride: Raman and Luminescence Investigations, Prospects for Use in Optoand Microelectronics. Publ. Center of Belarussian State University, Minsk.

[5] Shishonok, E.M., Steeds, J.W., Pysk, A.V., Mosunov, E.O., Abdullaev, O.R., Yakunin, A.S. and Zhigunov, D.M. (2012) Structural Studies of Rare-Earth Activated Cubic Boron Nitride Micropowders. Powder Metallurgy and Metal Ceramics, 50, 754-767. http://www.springerlink.com/openurl.asp?genre=article\&id=doi:10.1007/s11106-012-9386-5 http://dx.doi.org/10.1007/s11106-012-9386-5

[6] Shishonok, E.M. and Luhin, V.G. (2013) Evaluation of Type and Measure of Crystal Lattice Distortion of Cubic Boron Nitride Activated with Rare-Earth Elements. Proceedings of Belarussian State Technological University (BSTU): Phys.-Math. Sciences and Informatics, BSTU Publ. Center, Minsk, 6, 60-62.

[7] Bogdanov, S.P. (2008) Influence of Boron Impurities on the Crystal Structure of Cubic Boron. Glass Physics and Chemistry, 34, 218-223. http://dx.doi.org/10.1134/S108765960802017X

[8] Lang, A.R., Moore, M., Makepeace, A.P.W., Wierzchowski, W. and Welbourn, C.M. (1991) On the Dilatation of Synthetic Type Ib Diamond by Substitutional Nitrogen Impurity. Phyl. Trans. R. Soc. London. A, 337, 497-520.

[9] Abakumov, A.M. www.emat.ua.ac.be/xel2006/course\%20material/X-2006CD/Abakumov/LectureAbakumov.doc

[10] Weaver, M.L. http://bama.ua.edu/ mweaver/courses/MTE583/MTE\%20583_Class_17.pdf

[11] Shipilo, V.B., Shishonok, E.M., Korshunov, F.P. and Popelnuk, G.P. (1998) Method of Diamond Strengthening. Patent of Belarus No. 2282.

[12] LeClair, P.R. (2010) Structural Order and Disorder in Materials. 3.081 Module B Report, Group A, January 11.

[13] Darul, J. (2009) Thermal Instability of the Tetragonally Distorted Structure of Copper-Iron Materials. Kristallogr. Suppl., 30, 335-340. 